\title{
Overview of Liner Activities in Support of the International Forum for Aviation Research
}

\author{
M. G. Jones, $\quad$ D. M. Nark $†$ B. M. Howerton ${ }^{\ddagger}$ \\ NASA Langley Research Center, Hampton, VA 23681
}

\begin{abstract}
This paper provides an overview of liner research being conducted by members of the International Forum for Aviation Research (IFAR). The IFAR consists of representatives from a number of national research labs and is established to enable information exchange on aviation research activities. NASA has provided three challenges that explore different aspects of acoustic liner research, and will compile the results from each of the partners into a common database when the challenges are completed. The goal of the first challenge is to compare experimental methods applied at different national labs for acoustic liner evaluation, and to compare impedance eduction methodologies for uniform liners. The second challenge is to evaluate the ability of $3 \mathrm{D}$ propagation codes to predict the effects of spanwise variable-impedance liners on the acoustic pressure field. The third challenge is to explore different approaches to simultaneously educe the impedances for each zone of a three-zone liner. Each challenge is intended to be conducted as a blind test. Thus, only representative results achieved by the NASA Liner Physics Team for each of the three challenges are presented herein. When the results from all participants are available, the compiled results are intended to be provided in future reports.
\end{abstract}

\section{Introduction}

This paper provides an overview of liner research being conducted by members of the International Forum for Aviation Research (IFAR), and describes the contributions of the NASA Liner Physics Team to this activity. The IFAR consists of representatives from a number of national research labs and was established to enable information exchange on aviation research activities. In 2017, members of the IFAR Noise Working Group decided to include acoustic liner research as a topic for collaboration, and the NASA Langley Research Center was requested to serve as the topic lead. In support of this task, NASA proposed three challenges to explore different aspects of acoustic liner and duct aeroacoustics research. After some adjustments to account for varied interests, test capabilities, and computational resources of the IFAR members, these challenges were finalized as follows.

Challenge 1 is intended to allow for comparison of experimental methods. The goal is to gather data from multiple test rigs with simple liner configurations that can be built using additive manufacturing. The resultant data will be shared with each participant such that the dependence of the results on our respective liner manufacturing methods and data acquisition and analysis (e.g., impedance eduction) approaches can be assessed.

The goal of Challenge 2 is to evaluate multiple approaches to 3D aeroacoustic propagation via comparison with a single set of measured data, for which the geometry is sufficiently simple and the test rig is sufficiently small to minimize computational requirements. Three acoustic liners with uniform, spanwise symmetric, and spanwise asymmetric treatment, respectively, were previously tested ${ }^{11}$ in the NASA Grazing Flow Impedance Tube (GFIT, see Fig. 1). The impedance spectra as a function of Mach number, source sound pressure level (SPL), and frequency were educed for the uniform liner. Since the treated portion of each of these liners was identical (common facesheet and core geometry), these impedance values were assumed to apply to the treated portion of all three liners.

\footnotetext{
* Senior Research Scientist, Research Directorate, Structural Acoustics Branch, AIAA Associate Fellow.

${ }^{\dagger}$ Research Scientist, Research Directorate, Structural Acoustics Branch, AIAA Associate Fellow.

${ }^{\ddagger}$ Research Scientist, Research Directorate, Structural Acoustics Branch, AIAA Senior Member.
} 


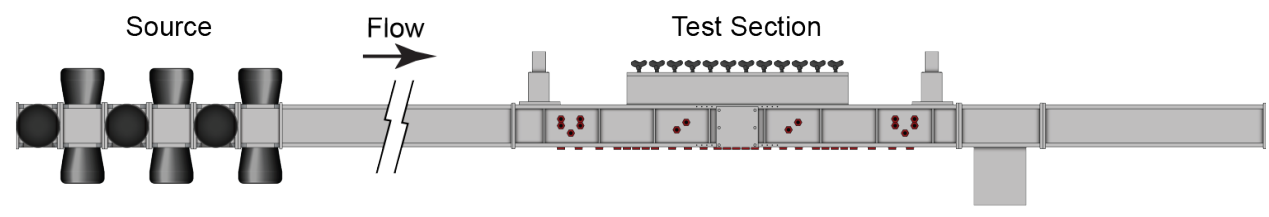

Figure 1: Sketch of NASA Grazing Flow Impedance Tube (GFIT).

These impedances were used as input to a $3 \mathrm{D}$ aeroacoustics propagation code to compute the sound field throughout the GFIT, in particular at each of the 95 microphone locations distributed along the walls of the duct. A comparison of the predicted and measured acoustic pressures at each of these locations was used to demonstrate the validity of the NASA 3D propagation code. The input data used in this study, including flow conditions, impedance spectra for the treated portions of each liner, GFIT geometry, and microphone locations, has been supplied to the IFAR participants, and they are currently using these inputs with their respective propagation codes to predict the acoustic pressure field within the GFIT. The results achieved with the different propagation codes will be compared with measured microphone responses and used to assess the relative strengths and weaknesses of different approaches.

Impedance eduction has been a topic of significant interest in the liner community over the last four decades.217 A number of approaches have been considered for this purpose, but have almost universally been implemented for uniform acoustic liners, i.e., liners for which the geometric properties are uniform over the entirety of their extent. As more advanced liner concepts are needed to meet increasingly stringent noise restrictions imposed by the International Civil Aviation Organization (ICAO) and the Federal Aviation Administration (FAA), there is a renewed interest in the exploration of multizone liners. The goal of Challenge 3 is to investigate different methods for studying axial multizone (multiple axial zones or segments) liners. Specifically, the desire is to establish the capability to simultaneously educe the impedance spectra for each zone. NASA will provide data acquired in the GFIT with a three-zone liner, and the challenge for each participant is to employ their respective multizone impedance eduction tools to determine the impedance spectra for each of the three zones.

The purpose of this paper is two-fold. The first goal is to provide a description of the three challenges, and to discuss the expected value to be gained from them. The second goal is to present NASA results for each challenge. It should be noted that these initial challenges were strongly influenced by NASA interests and thus represent a continuation of recent NASA research. It is hoped that these research activities will lead to other avenues of interest to the entire liner community. Section [II provides a detailed discussion of the three challenges, and Section [II] presents results achieved by NASA in support of each challenge. Concluding Remarks are provided in Section IV.

\section{Discussion of Liner Topic Challenges}

This section addresses each of the challenges proposed for the liner topic. An overview of each challenge is provided, along with a discussion of the background that led to the desire for its inclusion in this study. The goals for each challenge, along with the specific tasks to be completed, are also provided.

\section{A. Challenge 1}

\section{Overview and Background}

The main focus of this challenge is to support comparison of experimental methods as applied at a number of national research laboratories. There are a number of test rigs worldwide that are used for impedance eduction, each designed according to the needs of the individual organization. These test rigs tend to vary in geometry (e.g., cross-sectional dimensions and liner lengths), source capability (e.g., type and strength of acoustic source), measurement capability (e.g., type, number and location of microphones, implementation of Laser Doppler Velocimetry system), and flow control capability (e.g., maximum flow speed, flow profile, temperature control). Thus, any comparison of results achieved in different test rigs should include some 
discussion regarding the potential effects of these parameters on the reported results. Unfortunately, if the researcher has not planned for a collaborative study from the outset, some of these details are often omitted as they are not germane to the theme of the individual paper. In addition, everyone does not have access to the same materials and fabrication processes used to construct acoustic liners, especially the more novel configurations. This challenge is intended to address at least a portion of these difficulties. A secondary focus for this challenge is to compare impedance eduction methodologies for uniform liners.

\section{Goal}

The goal of this challenge is to gather data from multiple test rigs with a few simple liner configurations that can be readily built using additive manufacturing (3D printing). Each participant will fabricate versions of these liners to fit in their test rigs, and will acquire acoustic data (acoustic pressure, acoustic velocity, or both) using their data acquisition and analysis (e.g., impedance eduction) approaches. These data will be shared with all participants such that the dependence of the results on the different data acquisition and analysis approaches can be assessed.

\section{Task Description}

\section{NASA Tasks}

Two liners were selected for this investigation. Based on computer-aided design models of the liner geometry, each liner was built using a stereolithography (SLA) process whereby liquid resin (Accura 60) is photopolymerized using laser light to form the liner in 0.004"-thick layers. The perforated facesheet was integrated with the core while a solid backplate was used to seal the posterior of the liner. Sanding is performed on the front and back surfaces to ensure flatness for the backplate and GFIT mounting shoulders. The as-built geometry was verified via measurements for use in the prediction models.

The first liner is a uniform, single-degree-of-freedom (SDOF) liner, while the second is a two-zone liner (see Fig. 2). Each liner consists of a number of 0.4 "x0.4" square core chambers separated by 0.1 "-thick partitions, with an integrated conventional facesheet. The facesheet has a thickness of 0.034 ", hole diameters of $0.041 "$, and an open area ratio of 0.11 . The uniform liner has a constant core depth of 2.0 ", and the two-zone liner has two axial zones with unique core depths (3.0" depth for the 5.33"-long first zone and 2.0" depth for the 10.67'-long second zone).

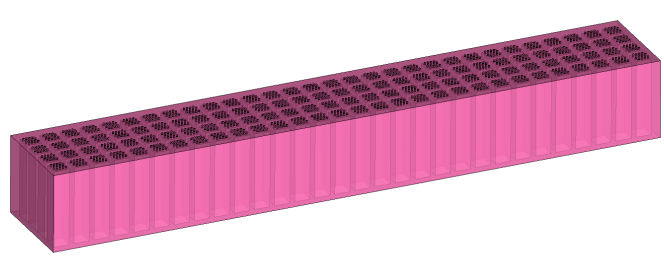

(a) Uniform liner.

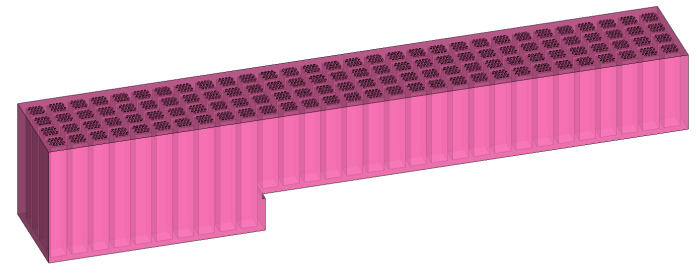

(b) Two-zone liner.

Figure 2: Sketches of uniform and two-zone liners used for Challenge 1.

\section{IFAR Participant Tasks}

The drawings supplied by NASA are for samples with active areas (treated portion) of 2.0"-wide by 16.0"long. Each participant will modify the drawings to fit their respective test rigs. To the extent possible, the chamber dimensions will be held constant, but the number of chambers will be modified (in either the axial or spanwise dimensions) to achieve the required liner dimensions suitable for the specific test rig. These 
modifications will be documented such that the effects of these changes can be investigated. An attempt will also be made to understand the effects of using different additive manufacturing techniques and materials.

Next, each liner will be tested in the participant's test rig, and the results (acoustic pressure, velocity, or both) will be provided to each partner. This will allow each participant to compare results with similar (not identical) liners mounted in a number of test rigs. The expectation is that this collaboration will help to understand the difference between results attained in the respective test rigs. Also, each participant will educe the impedance for the uniform liner. This may be done with direct or indirect methods. Although the results may differ somewhat, the comparison should nevertheless be favorable.

\section{B. Challenge 2}

\section{Overview and Background}

As the noise requirements for commercial aircraft become increasingly stringent, the need to employ more novel and complex liner configurations continues to grow. One concept that is of interest is variableimpedance liners, i.e., liners for which the impedance varies in either the axial and/or circumferential dimensions. Axial variations have been studied extensively (often labeled as multisegment or multizone liners, see Challenges 1 and 3), whereas less attention has been given to circumferential (spanwise) variations. One possible reason is that axial impedance variation can be studied (to a large extent) with 2D propagation codes, whereas spanwise impedance variation requires the use of more computationally intensive 3D propagation codes. In addition, there are not many validation cases for which the geometry is sufficiently simple to allow cross-validation of multiple codes and for which the data is readily available. Thus, the main focus of the second challenge is to support evaluation of $3 D$ aeroacoustic propagation codes.

At the 2017 AIAA/CEAS Aeroacoustics Conference, NASA presented 11 comparisons of predicted and measured acoustic pressure profiles for three additively manufactured acoustic liners (Figure 3). The first is a uniform impedance, SDOF liner. The second is a symmetric spanwise variable-impedance liner, and the last is an asymmetric spanwise variable-impedance liner. More details regarding the specific configurations can be found in that paper. Data were acquired in the GFIT with each of these three liners, to include acoustic pressures at 95 microphone locations distributed along all four walls of the GFIT test section and information regarding the test environment (static temperature, static pressure, centerline and average Mach number, etc.). For most test conditions, the predicted results (SPL and phase) attained with three 3D propagation codes currently used by NASA were shown to compare favorably with the measured data. However, each code was shown to perform best for different test conditions.

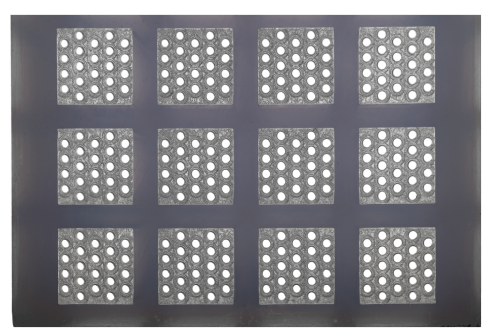

(a) Uniform liner.

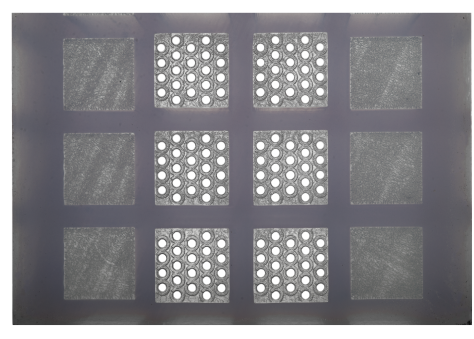

(b) Spanwise symmetric liner.

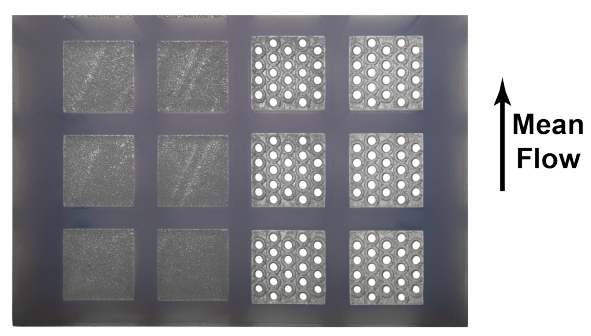

(c) Spanwise asymmetric liner.

Figure 3: Partial view of liners used for Challenge 2.

\section{Goal}

The goal of this challenge is to evaluate multiple approaches to 3D propagation via comparison to this NASA set of measured data, for which the geometry is sufficiently simple and the test rig is sufficiently small to minimize computational requirements. This will allow evaluation of the relative strengths and weaknesses of the different approaches. 


\section{Task Description}

NASA Tasks

Test data acquired in the GFIT with each of the three liners described above have been provided to the IFAR participants. This data includes the test environment details, GFIT microphone locations, source and termination conditions (i.e., the termination impedance of the GFIT), and the surface impedance spectra for each spanwise zone of these liners. It does not include the measured acoustic pressures (SPL and phase) at each of the microphone locations, as this is intended to be a blind test. Instead, that information will be provided after the participants conclude their predictions.

IFAR Participant Tasks

Each participant will use their respective $3 \mathrm{D}$ propagation codes to predict the acoustic pressures at each GFIT microphone location. They are requested to provide these predicted acoustic pressures, along with the underlying assumptions used in their 3D propagation code(s), for comparison with the corresponding results from other participants. They are also requested to provide timing and CPU requirements needed to complete their analysis. All of the predicted and measured acoustic pressures will be gathered into a single database for comparison by all participants.

\section{Challenge 3}

\section{Overview and Background}

Axial multizone (axially segmented) liners have been studied for many years, but their implementation in commercial aircraft has generally been due to other constraints (e.g., need for space to contain ancilliary systems) rather than acoustic potential. As noise restrictions become more stringent, and as the amount of space for acoustic liners is reduced, multizone liners may be of increased interest as they are becoming simpler to manufacture and may offer some additional noise-reduction capability. However, their use would require implementation of methods to educe acoustic properties (e.g., the impedance) of each liner zone.

There are at least three ways in which the impedances of each axial zone can be determined. One is to employ local measurements (e.g., the Dean in situ method ${ }^{[18}$ ) to educe the impedance for each zone. The second is to build uniform liners that correspond to the different zones of the multizone liner, and then to use standard uniform-impedance methods to educe the impedance for each liner (and therefore, for each zone of the multizone liner). The focus of this challenge is to explore a third option, namely methods that simultaneously educe the impedances for multiple axial zones.

\section{Goal}

The goal for this challenge is to assess the relative strengths and weaknesses of different methods for studying multizone liners. Results will be compared on the basis of accuracy and computational requirements.

\section{Task Description}

\section{NASA Tasks}

A liner has been designed that consists of three axial zones (segments) with lengths of 6.0", 7.2", and 8.8", respectively, for a total liner length of 22". It was fabricated using the additive manufacturing method described earlier in Section 3 and tested in the GFIT. Three uniform liners were also fabricated, with geometric properties that correspond to each of the axial zones of the three-zone liner.

Data were acquired at three centerline Mach numbers (0.0, 0.3, and 0.5) and two source sound pressure levels $(120$ and $140 \mathrm{~dB}$ ) for each of these liners. These data, together with test environment details and GFIT microphone locations, were recently provided to IFAR participants.

A direct method based on the Kumaresan and Tufts algorithm was used to educe the impedance spectra for each of the uniform liners, and an indirect method based on the convected Helmholtz equation was used to simultaneously educe the impedance spectra for each zone of the three-zone liner. These results will be used for comparison with those attained by each IFAR participant with their respective multizone impedance eduction methods. 
IFAR Participant Tasks

Each participant will utilize their respective tools (or in some cases, develop new ones) to analyze the GFIT data, and will educe impedance spectra for each of the three axial zones. Each participant is requested to provide their respective educed impedance spectra for each axial zone for comparison with the results attained by other participants. They will also provide details regarding their impedance eduction approaches, to include timing and CPU requirements. It should be noted that the NASA data was only recently provided to the IFAR participants. Thus, initial comparisons of the results from the respective participants will be provided in future reports.

\section{Summary of NASA Results}

\section{A. Challenge 1}

A uniform liner and a two-zone liner were fabricated according to the requirements listed in Section II] and were tested in the GFIT. Data were acquired at two flow conditions (Mach 0.0 and 0.3) and two source levels $(120$ and $140 \mathrm{~dB})$. The resultant data were used to investigate a multizone impedance eduction approach 19 (see Section II C). For the purposes of this challenge, the acoustic pressure data acquired at 95 microphone locations in the GFIT, along with the test environment information will be included in the comparison database based on collaboration with the respective IFAR participants.

Figure 4 provides a sampling of acoustic pressure profiles (SPL portion) acquired in the GFIT with the uniform and two-zone liners. In each case, the liner extends over the axial range of $\left\{8^{\prime \prime}, 24^{\prime \prime}\right\}$. The results presented herein are confined to mid-range frequencies for which the largest effects (greatest attenuation) were observed at Mach 0.3 and a source level of $140 \mathrm{~dB}$.

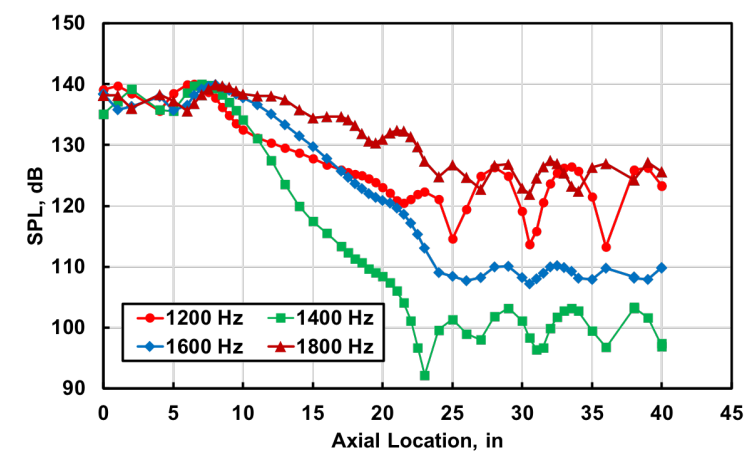

(a) Uniform liner.

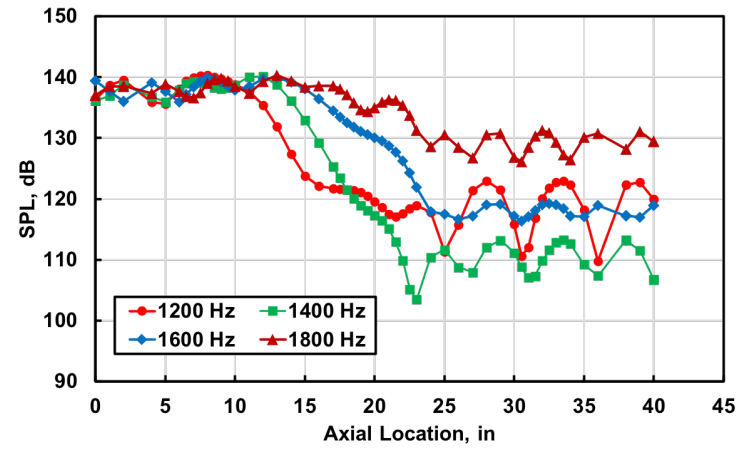

(b) Two-zone liner.

Figure 4: Axial sound pressure level profiles. Mach 0.3, $140 \mathrm{~dB}$ source.

The earlier paper ${ }^{19}$ provides impedances educed for each of these liners using methods designed for uniform and two-zone liners. Results for the Mach 0.3 flow condition and a source level of $140 \mathrm{~dB}$ are presented in Figure 5. They are confined to the frequency range of 400 to $2400 \mathrm{~Hz}$, as higher-order modes are cut on at the next higher frequency $(2600 \mathrm{~Hz})$. Note that reactances educed for the 2" depth treatment, whether they are for the uniform liner or the downstream zone of the two-zone liner, compare favorably over the entire frequency range. The corresponding comparison of resistances is also favorable for frequencies where significant attenuation was achieved, but degrades at frequencies where the attenuation is reduced.

The two-zone impedance eduction method has recently been upgraded ${ }^{20}$ to allow for more zones and to account for an improved understanding of the mean flow effects on impedance eduction. ${ }^{21}$ This method will be used for the final impedance eduction comparisons with IFAR partners. A more thorough review of the IFAR results for Challenge 1, including those contributed by NASA, will be provided in a future report. 


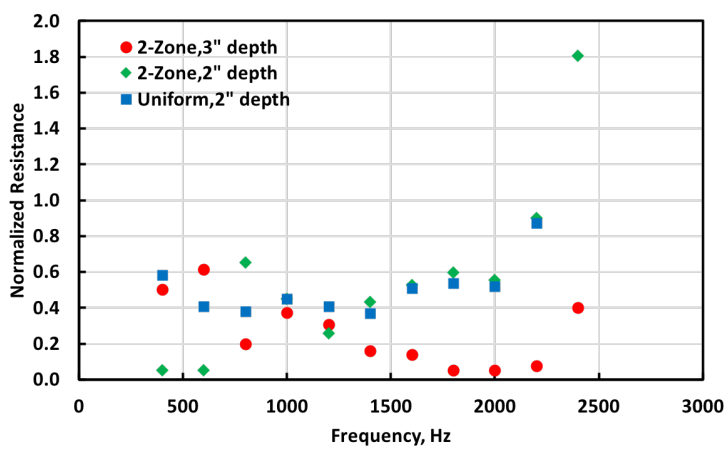

(a) Normalized resistance.

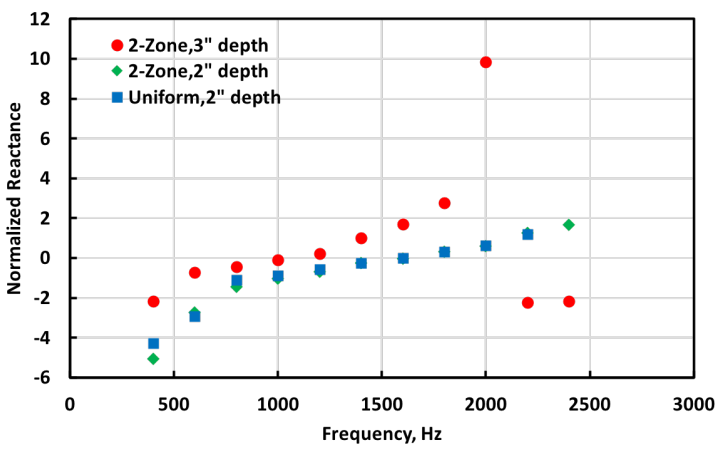

(b) Normalized reactance.

Figure 5: Educed impedances for Challenge 1 liners. Mach 0.3, $140 \mathrm{~dB}$ source.

\section{B. Challenge 2}

NASA previously presented ${ }^{1}$ results from a study to evaluate the uniform, spanwise symmetric, and spanwise asymmetric liners described in Section IIIB. Data acquired in the GFIT were compared with predictions generated using three 3D aeroacoustic propagation codes; CHE ${ }^{[2]}$ CDL $\stackrel{23}{, 23}$ and COMSOL ${ }^{[24}$ Each of these codes solves the convected Helmholtz equation and, for the purposes of this study, employs a uniform flow profile and a plane-wave source. Both CHE and COMSOL solve a second order partial differential equation using a conventional Galerkin finite element method, and include effects of reflections at the leading and trailing edges of the liner and at the duct termination. The CDL code utilizes a parabolic approximation to the convected Helmholtz equation, and neglects reflections due to impedance discontinuities at the leading and trailing edges of the liner. It also assumes the duct termination is anechoic. Additional details are provided in the references, and will thus not be repeated here for the sake of brevity.

A portion of the results from the 2017 report are presented in Figures 6 and 7, at frequencies (1000 and $1100 \mathrm{~Hz}$ ) where a healthy amount of attenuation was achieved. In general, comparisons between measured and predicted SPL profiles are quite favorable. However, since the amount of treatment (number of active chambers) is identical for the symmetric and asymmetric liners, a 2D propagation code would predict them to produce identical SPL profiles. Instead, the divergence between the respective profiles provides clear indication of the need for $3 \mathrm{D}$ propagation codes.

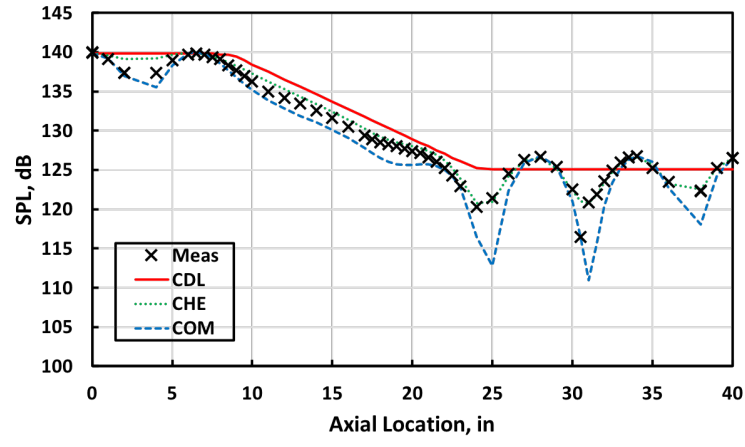

(a) $1000 \mathrm{~Hz}$

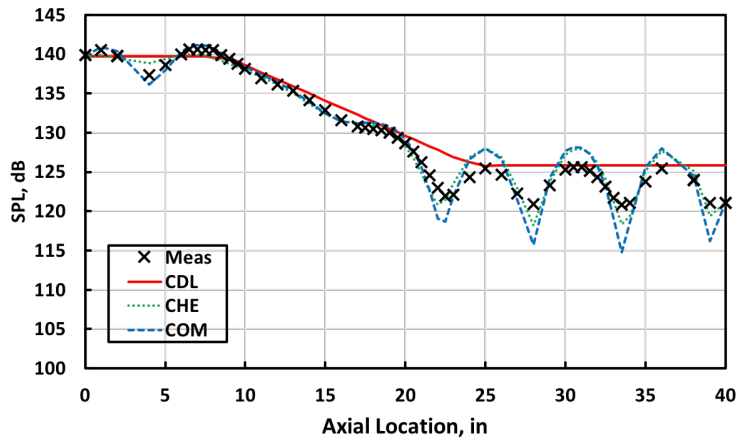

(b) $1100 \mathrm{~Hz}$.

Figure 6: Comparison of measured and predicted sound pressure level profiles for spanwise symmetric liner. Mach $0.3,140 \mathrm{~dB}$ source.

A more thorough review of the full set of data demonstrates that each code has particular regimes where 
the comparison with measured data is less favorable, but generally each of the codes is well suited for this type of analysis. Overall, the SPL profiles for the two spanwise variable-impedance liners are very similar when the total attenuation is minimal, but they diverge as the attenuation increases. This divergence of SPL profiles is present in the Mach 0.3 results, but is more evident at Mach 0.0 (see Fig. 8) because these liners are tuned (via the facesheet geometry) for maximum attenuation at the no-flow condition.

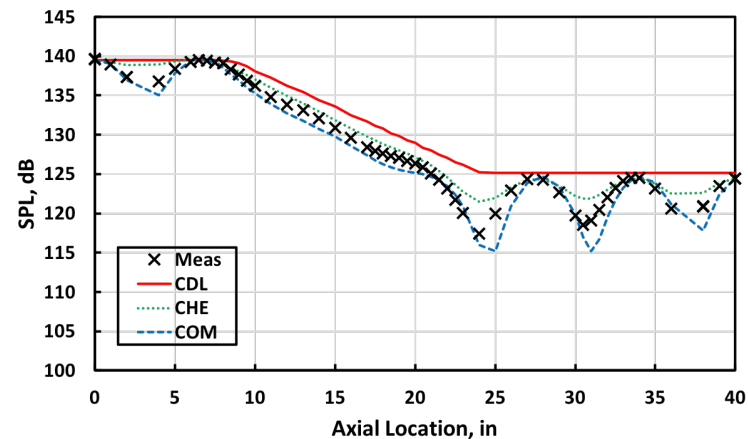

(a) $1000 \mathrm{~Hz}$

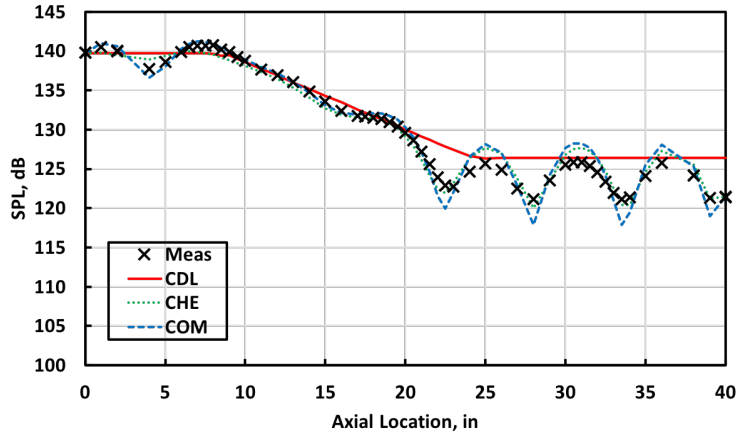

(b) $1100 \mathrm{~Hz}$.

Figure 7: Comparison of measured and predicted sound pressure level profiles for spanwise asymmetric liner. Mach $0.3,140 \mathrm{~dB}$ source.

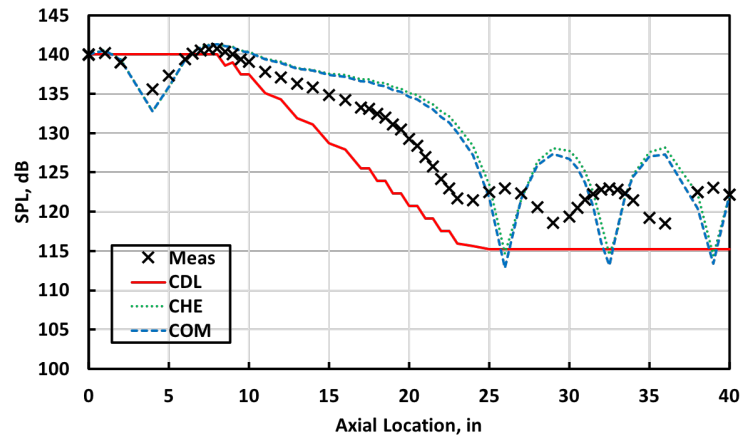

(a) Asymmetric liner.

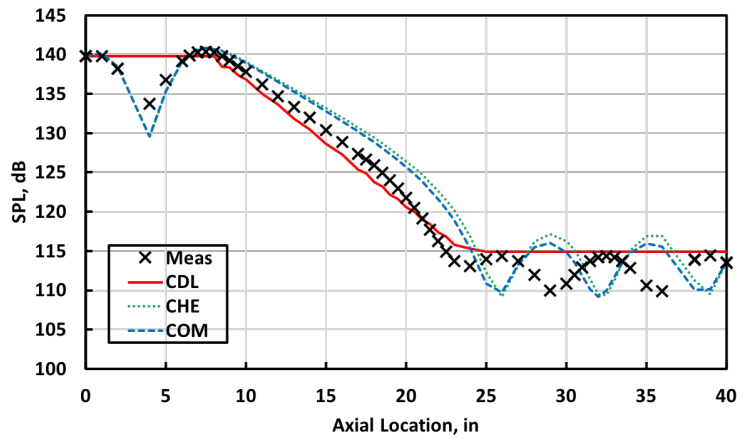

(b) Symmetric liner.

Figure 8: Comparison of measured and predicted sound pressure level profiles for spanwise variableimpedance liners. Mach 0.0, $1050 \mathrm{~Hz}, 140 \mathrm{~dB}$ source.

\section{Challenge 3}

As noted earlier, design of the three-zone liner has recently been completed. Challenge 3 is intended to be a blind test, where each participant will educe the impedance of each zone based on the measured acoustic pressure data. Thus, the specific geometry of the liner cannot be shown herein. However, it is similar in design to the two-zone liner used by NASA ${ }^{19}$ for an earlier study. The approach used in that study to educe the impedances for a two-zone liner was based on extension of an existing uniform-liner impedance eduction technique,$\frac{25}{25}$ and was demonstrated to successfully educe the impedance for both zones. This approach has been extended to account for additional zones, and will be used to study the three-zone liner.

Figure 9 provides acoustic pressure profiles (sound pressure level and phase) measured with the three-zone liner mounted in the GFIT. These data were acquired for a centerline Mach number of 0.3 and a source SPL of $140 \mathrm{~dB}$, and are presented for frequencies of 1200, 1400, 1600, and $1800 \mathrm{~Hz}$. There are distinct changes 
in the SPL profiles [Fig. 9(a)] over the three zones, whereas the phase profiles [Fig. 9(b)] show less zone dependence.

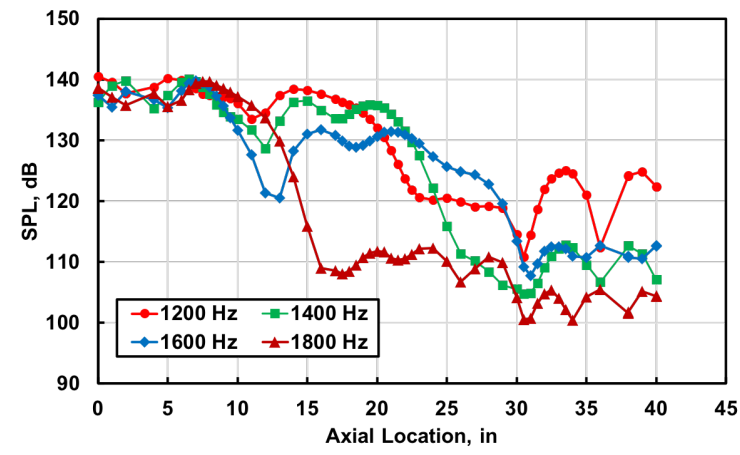

(a) Sound pressure level.

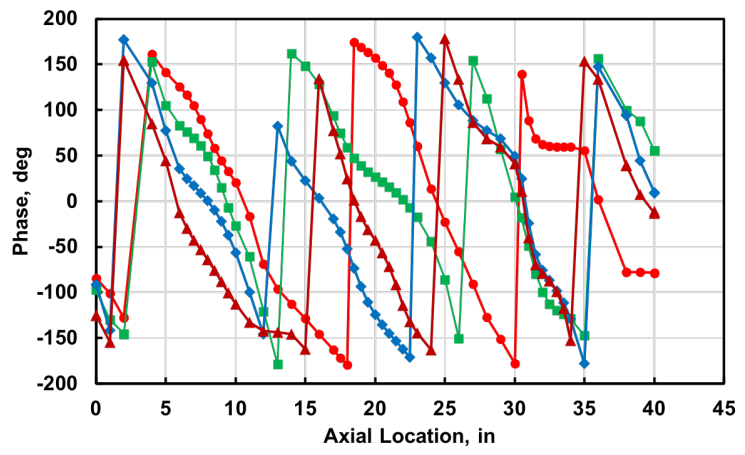

(b) Phase.

Figure 9: Measured sound pressure and phase profiles for three-zone liner. Mach 0.3, $140 \mathrm{~dB}$ source.

\section{Concluding Remarks}

This paper presented an overview of liner research being conducted by members of the International Forum for Aviation Research (IFAR), and describes the contributions of the NASA Liner Physics Team to this activity. Research is currently targeted toward the completion of three challenges. The first challenge seeks to support comparison of experimental methods as applied at different national labs, and to compare impedance eduction methodologies for uniform liners. The second evaluates the ability of 3D propagation codes to predict the effects of spanwise variable-impedance liners on the acoustic pressure field. The last explores different approaches to simultaneously educe the impedances for each zone of a three-zone liner.

Each of the three challenges are intended to be conducted by the IFAR partners as blind tests. Thus, for now, only representative results achieved by the NASA Liner Physics Team for each of the three challenges were presented. When the results from all participants are available (some of which will be presented in companion papers at this conference), the compiled results are intended to be provided in future reports.

\section{Acknowledgements}

This work was funded by the Advanced Air Transport Technology Project of the NASA Advanced Air Vehicles Program. The support of Martha Brown and Max Reid for the acquisition of GFIT data and Robert Andrews of Langley's Additive Manufacturing Center for support related to additive manufacturing is gratefully acknowledged.

\section{References}

${ }^{1}$ Jones, M. G., Watson, W. R., Nark, D. M., and Schiller, N. H., "Evaluation of Spanwise Variable Impedance Liners with Three-Dimensional Aeroacoustics Propagation Codes," AIAA Paper 2017-3021, June 2017.

${ }^{2}$ Armstrong, D. L., Beckemeyer, R. J., and Olsen, R. F., "Impedance Measurements of Acoustic Duct Liners With Grazing Flow," Paper presented at 87th Meeting of the Acoustical Society of America 1999-1864, 1974.

${ }^{3}$ Watson, W. R., Jones, M. G., and Parrott, T. L., "Validation of an Impedance Eduction Method in Flow," AIAA Journal, Vol. 37, No. 7, July 1999, pp. 818-824.

${ }^{4}$ Jones, M. G., Watson, W. R., Tracy, M. B., and Parrott, T. L., "Comparison of Two Waveguide Methods for Educing Liner Impedances in Grazing Flow," AIAA Journal, Vol. 42, No. 2, Februrary 2004, pp. 232-240.

${ }^{5}$ Jones, M. G., Watson, W. R., and Parrott, T. L., "Benchmark Data for Evaluation of Aeroacoustic Propagation Codes with Grazing Flow," AIAA Paper 2005-2853, May 2005.

${ }^{6}$ Busse, S., Richter, C., Thiele, F. H., Heuwinkel, C., Enghardt, L., Rohle, I., Michel, U., Ferrante, P., and Scofano, 
A., "Impedance Deduction Based on Insertion Loss Measurements of Liners under Grazing Flow Conditions," AIAA Paper 2008-3014, May 2008.

${ }^{7}$ Watson, W. R. and Jones, M. G., "Comparison of Convected Helmholtz and Euler Model for Impedance Eduction in Flow," AIAA Paper 2006-2643, May 2006.

${ }^{8}$ Jing, X., Peng, S., and Sun, X., "A Straightforward Method for Wall Impedance Eduction in a Flow Duct," Journal of the Acoustical Society of America, Vol. 124, No. 1, July 2008.

${ }^{9}$ Elnady, T., Boden, H., and Elhadidi, B., "Validation of an Inverse Semi-Analytical Technique to Educe Liner Impedance," AIAA Journal, Vol. 47, No. 12, December 2009, pp. 2836-2844.

${ }^{10}$ Eversman, W. and Gallman, J. M., "Impedance Eduction with an Extended Search Procedure," AIAA Paper 2009-3235, May 2009.

${ }^{11}$ Renou, Y. and Auregan, Y., "Failure of the Ingard-Myers Boundary Condition for a Lined Duct: An Experimental Investigation," Journal of the Acoustical Society of America, Vol. 130, No. 1, July 2011, pp. 52-60.

${ }^{12}$ Busse-Gerstengarbe, S., Bake, F., Enghardt, L., and Jones, M. G., "Comparative Study of Impedance Eduction Methods, Part 1: DLR Tests and Methodology," AIAA Paper 2013-2124, May 2013.

${ }^{13}$ Jones, M. G., Watson, W. R., Howerton, B. M., and Busse-Gerstengarbe, S., "Comparative Study of Impedance Eduction Methods, Part 2: NASA Tests and Methodology," AIAA Paper 2013-2125, May 2013.

${ }^{14}$ Jones, M. G. and Watson, W. R., "Validation of an Improved Experimental Method for Use in Impedance Eduction," AIAA Journal, Vol. 51, No. 1, January 2013, pp. 186-199.

${ }^{15}$ Watson, W. R. and Jones, M. G., "A Comparative Study of Four Impedance Eduction Methodologies Using Several Test Liners," AIAA Paper 2013-2274, May 2013.

${ }^{16}$ Watson, W. R., Carpenter, M. H., and Jones, M. G., "Performance of Kumaresan and Tufts Algorithm in Liner Impedance Eduction with Flow," AIAA Journal, Vol. 53, No. 4, April 2015, pp. 1091-1102.

${ }^{17}$ Weng, C., Schulz, A., Ronneberger, D., and Bake, F., "Impedance Eduction in the Presence of Turbulent Shear Flow Using the Linearized Navier-Stokes Equations," AIAA Paper 2017-3182, June 2017.

${ }^{18}$ Dean, P. D., "An In Situ Method Of Wall Acoustic Impedance Measurement In Flow Ducts," Journal of Sound and Vibration, Vol. 34, No. 1, 1974, pp. 97-130.

${ }^{19}$ Jones, M. G., Watson, W. R., Nark, D. M., and Howerton, B. M., "Impedance Eduction for Multisegment Liners," AIAA Paper 2018-3441, June 2018.

${ }^{20}$ Jones, M. G., , Nark, D. M., and Watson, W. R., "Evaluation of a Multizone Impedance Eduction Method," AIAA Paper 2019-xxxx, May 2019.

${ }^{21}$ Nark, D. M., Jones, M. G., and Piot, E., "Assessment of Axial Wavenumber and Mean Flow Uncertainty on Acoustic Liner Impedance Eduction," AIAA Paper 2018-3444, June 2018.

${ }^{22}$ Watson, W. R. and Jones, M. G., "Impedance Eduction in 3D Sound Fields with Peripherally Varying Liners and Flow," AIAA Paper 2015-2228, June 2015.

${ }^{23}$ Nark, D. M. and Jones, M. G., "Broadband Liner Optimization for the Source Diagnostic Test Fan," AIAA Paper 2012-2195, June 2012.

24"COMSOL Acoustic Module User's Guide," v5.1, 2015.

${ }^{25}$ Watson, W., Jones, M., and Parrott, T., "A Quasi-3-D Theory for Impedance Eduction in Uniform Grazing Flow," AIAA Paper 2005-2848, May 2005. 